\title{
Clinical Significance of an Unusual Variation Anomalous additional belly of the sternothyroid muscle
}

"Senthil Murugan M., ${ }^{1}$ Sudha R., ${ }^{1}$ Rajesh Bhargavan"

سنثل مرجان، سودا، راجييش بهراجافان

\begin{abstract}
The infrahyoid muscles are involved in vocalisation and swallowing; among these, the sternothyroid muscle is derived from the common primitive sheet. The improper differentiation of this muscle may therefore result in morphological variations. We report an unusual variation found during the dissection of a 65-year-old male cadaver at the Sri Manakula Vinayagar Medical College, Madagadipet, Pondicherry, India, in 2015. An anomalous belly of the right sternothyroid muscle was observed between the internal jugular (IJ) vein and the internal carotid artery with an additional insertion into the tympanic plate and petrous part of the temporal bone and the presence of a levator glandulae thyroideae muscle. The anomalous muscle may compress the IJ vein if it is related to the neurovascular structures of neck; hence, knowledge of variations of the infrahyoid muscles can aid in the evaluation of IJ vein compression among patients with idiopathic symptoms resulting from venous congestion.
\end{abstract}

Keywords: Neck Muscles; Thyroid Gland; Cervical Plexus; Jugular Veins; Case Report; India.

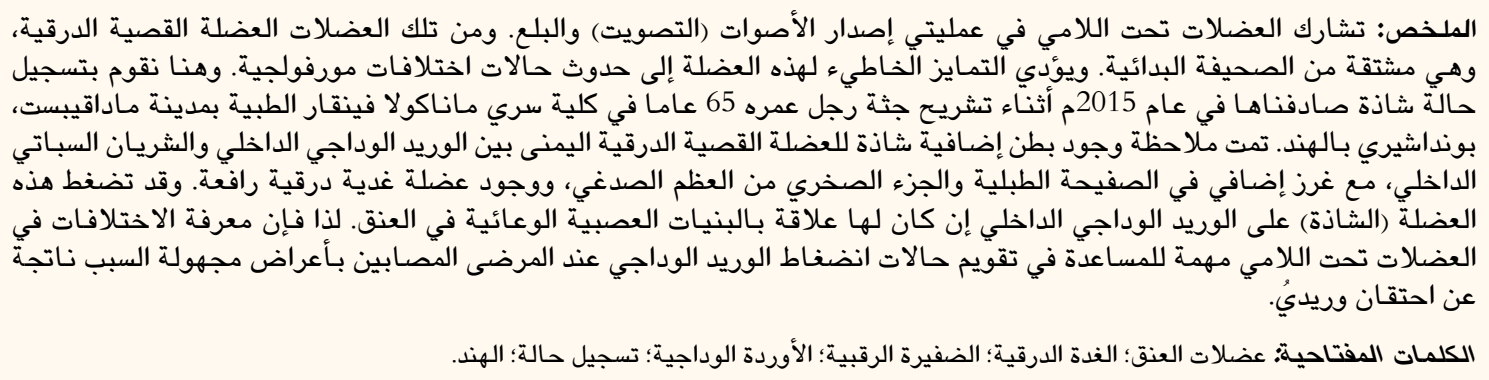

$\mathrm{I}$

N THE VENTROLATERAL REGION OF THE NECK, the muscles are arranged in three strata: the subcutaneous, superficial and deep brachiocephalic layers. These three layers are derived through the stratification of a single primitive muscle sheet, which can still be found in some lower vertebrates, and retains direct continuity with the abdominal part of the ventral muscle. ${ }^{1}$ The sternothyroid (ST) muscle is a part of the deep brachiocephalic layer and lies deep to as well as medial to the sternohyoid muscle; it originates from the posterior surface of the manubrium sterni and the posterior margin of the first costal cartilage, passing into the oblique line of the thyroid cartilage. ${ }^{2}$ The ST muscle may be in two layers or many fascicles; among them, the lateral layer may end in the fascia colli. ${ }^{3,4}$ A levator glandulae thyroideae (LGT) fibrous or fibromuscular band may descend from the inferior margin of the hyoid bone to the lobes, isthmus or pyramidal lobe of the thyroid gland. ${ }^{4}$ A LGT muscle may be present alongside variations of the infrahyoid muscles. ${ }^{5}$

\section{Case Report}

A regular anatomy dissection session of a 65-yearold male cadaver was performed at the Sri Manakula Vinayagar Medical College, Madagadipet, Pondicherry, India, in 2015. During this dissection, an anomalous right ST muscle was found. The muscle originated from the posterior surface of the manubrium sterni at the medial end of the costal cartilage of the first rib. It divided into a medial and a lateral slip approximately $2.7 \mathrm{~cm}$ distal to its origin. The medial slip was inserted superiorly to the oblique line of the thyroid cartilage; however, the lateral slip ascended and some of its fibres were attached to the fascial sheath of the great vessels of the neck, also known as the carotid sheath [Figure 1]. The muscle terminated between the internal jugular (IJ) vein and internal carotid (IC) artery as well as the emerging glossopharyngeal and hypoglossal nerves [Figure 2]. It was attached to the lower sharp edge of the tympanic plate of the temporal bone and also to the bony ridge 


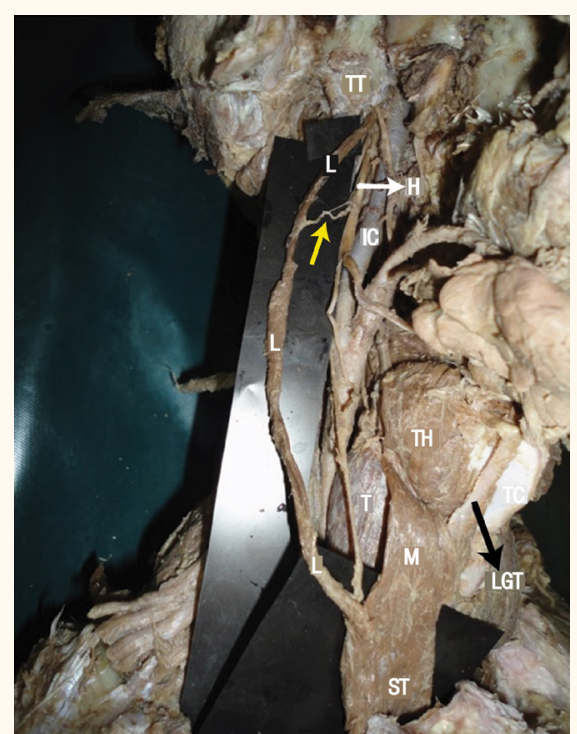

Figure 1: Photograph of a deep dissection of the right side of the neck of a 65-year-old male cadaver showing the sternothyroid muscle divided into two slips. The medial slip passed above the oblique line on the lamina of the thyroid cartilage. The lateral slip passed into the tympanic plate of temporal bone and to the bony ridge of the petrous part of the temporal bone between the carotid canal and jugular fossa. A nerve twig from C1 supplied the lateral slip (yellow arrow). Note the location of the hypoglossal nerve (white arrow) and levator glandulae thyroideae muscle (black arrow).

$T T=$ tympanic plate of temporal bone; $L=$ lateral slip; $H=$ hypoglossal nerve; $I C=$ internal carotid artery; $T H=$ thyrohyoid muscle; $T C=$ thyroid cartilage; $T=$ thyroid gland; $M=$ medial slip; $L G T=$ levator glandulae thyroideae $m u s c l e ; S T=$ sternothyroid muscle.

of the petrous part of the temporal bone between the carotid canal and jugular fossa. The medial bundle was supplied by a branch of the ansa cervicalis nerves, whereas the lateral bundle was supplied by branches of the ansa cervicalis (C2 and C3) nerves as well as the hypoglossal nerve $(\mathrm{C} 1)$ before it formed the descendens hypoglossi.

An LGT muscle was also found on the same side, arising from the hyoid bone and passing into the false capsule on the anterior margin of the right lobe of the thyroid gland. Since the anomalous lateral belly of the ST muscle was between the great vessels of the neck, this could have caused compression of the IJ vein and potentially resulted in venous stasis of its formative tributary, the sigmoid sinus. The cranial cavity was therefore opened to examine the variation in the size of the sigmoid sulcus on the right side. An osseous bridge was observed over the right sigmoid sulcus in the posterior cranial fossa connecting the angulation of the anterior lip with the posterior lip. The sigmoid sulcus of the sinus was significantly larger and deeper on the right side of the posterior cranial fossa [Figure 3].

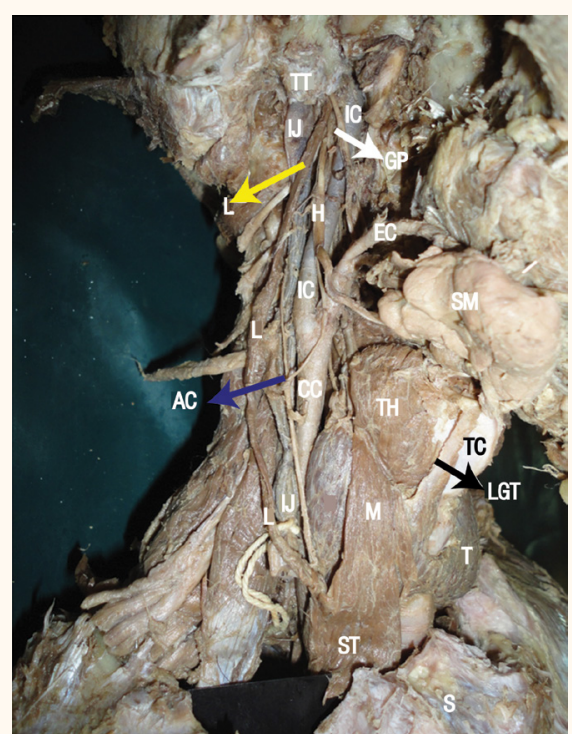

Figure 2: Photograph of a deep dissection of the right side of the neck of a 65 -year-old male cadaver showing an anomalous lateral belly of the right sternothyroid muscle. The terminal part of the belly was located between the internal jugular vein and the internal carotid artery and ascended between the hypoglossal and glossopharyngeal (white arrow) nerves. Note the location of the lateral slip (yellow arrow), ansa cervicalis (blue arrow) and levator glandulae thyroideae muscle (black arrow).

$T T=$ tympanic plate of temporal bone; $I C=$ internal carotid artery; II = internal jugular vein; $G P=$ glossopharyngeal nerve; $H=$ hypoglossal nerve; $E C=$ external carotid artery; $S M=$ submandibular salivary gland; $L=$ lateral slip; $C C=$ common carotid artery; $A C=$ ansa cervicalis; $T H=$ thyrohyoid muscle;

$T C=$ thyroid cartilage; $L G T=$ levator glandulae thyroideae muscle; $T$ = thyroid gland; $M=$ medial slip; $S T$ = sternothyroid muscle; $S=$ sternum.

\section{Discussion}

Pre-muscle tissue (approximately $7 \mathrm{~mm}$ in length) appears in the cervical region of very young embryos as a result of mesenchymal condensation and with the contribution of myoblasts of the cervical hypomere (e.g. the hypaxial mesoderm). ${ }^{6}$ The $T B X 1$ gene regulates the myogenic differentiation and cellular fate within the mesoderm. ${ }^{7}$ This gene codes for the T-box protein and is found on the $\mathrm{q}$ arm of chromosome 22 at position 11.21. Inactivation or deletion of this gene can lead to serious alterations in the morphology of neck muscles. ${ }^{7,8}$ Mesenchymal condensation later develops into a distinct band of infrahyoid pre-muscle tissue extending to either side, from the base of the tongue caudolaterally towards the tip of the first rib. Cleavage of this tissue thus forms the most ventral muscles of the neck which are supplied by the ramus of the descendens hypoglossi. ${ }^{6}$ Initially, the infrahyoid pre-muscle band divides into deep and superficial layers, after which the superficial sheath then divides into inner and outer muscles; the inner muscle eventually forms the sternohyoid muscle and the 


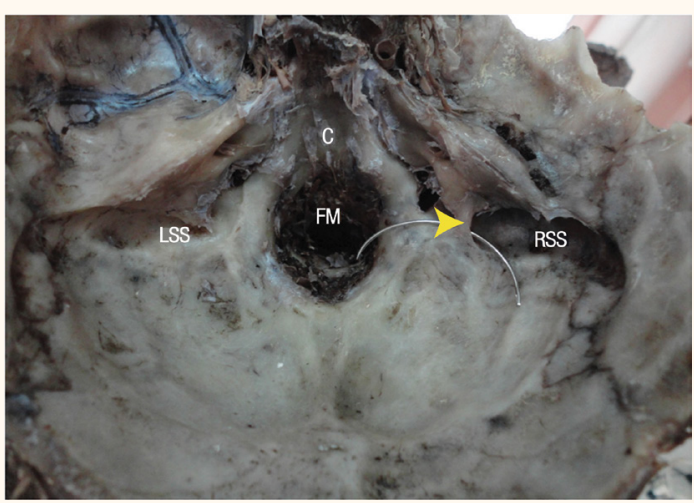

Figure 3: Photograph of the posterior cranial fossa of a 65-year-old male cadaver. The right sigmoid sulcus (RSS) was larger and deeper compared to the left sigmoid sulcus. An osseous bridge connected the anterior and posterior lips of the RSS (arrowhead). $C=$ clivus; $F M=$ foramen magnum; $L S S=$ left sigmoid sulcus; $R S S=$ right sigmoid sulcus .

lower part of the outer muscle forms the omohyoid muscle. ${ }^{9}$ The deep stratum of the undifferentiated muscle mass becomes the ST and thyrohyoid muscles. Since the pre-muscle mass extends from the base of the tongue to the tip of first rib, improper cleavage and differentiation may lead to variations of the derivative muscles.

To the best of the authors' knowledge, the variation of the ST muscle observed in the present case is unique and has not been previously reported. Among 36 cadavers with muscular variations reported in previous research, one had a duplicate ST muscle with the additional slip arising from the first costal cartilage and the costoclavicular ligament, joining the normal one halfway up the neck. ${ }^{10}$ In an earlier similar observation by the same author, the abnormal slip (known as the costo-fascialis cervicalis) terminated in the cervical fascia under the sternocleidomastoid muscle. ${ }^{10}$ Humphry reported that the ST muscle can be double, either wholly, partially or divided into two lateral portions running parallel with one another. ${ }^{1}$ However, in the present case, the lateral belly was attached to the carotid sheath as well as to the bony ridge between the jugular fossa and the lower opening of the carotid canal.

In the present case, the cranial half of the anomalous lateral belly of the ST muscle passed between the IJ vein and the IC artery for a short distance before its insertion. These neurovascular links are of great clinical value; other reports have indicated that variations of the omohyoid muscle resulted in compression of the IJ vein, which could in turn hinder venous return from the head and neck. ${ }^{11-13}$ In addition, the IJ vein can be compressed by other abnormal neck muscles, including variations of the sternocleidomastoid and digastric muscles. ${ }^{11,12,14-16}$ Compression of the IJ vein may lead to IJ vein thrombosis and cause an embolism in the pulmonary artery. ${ }^{17}$ In the current case, the anomalous lateral belly of the ST muscle may have compressed the IJ vein and caused thrombosis; however, this cannot be known for sure. In addition, the anomalous belly of the ST muscle lay between the glossopharyngeal and hypoglossal nerves near its termination. This may have compressed the nerves related to this region, potentially resulting in unilateral palsy. As such, it is possible that the cadaver suffered from Collet-Sicard syndrome; this condition involves the IX to XII cranial nerves and can be caused by idiopathic IJ vein thrombosis. ${ }^{18}$

Sigmoid sinus enlargement can cause pulsatile tinnitus. ${ }^{19}$ In the current case, the sulcus of the right sigmoid sinus was significantly enlarged, perhaps due to the compression of the IJ vein by the anomalous lateral belly on the right side. The usual course of the sigmoid sulcus is vertical on the mastoid section of the temporal bone, followed by anteromedial in the jugular process of the occipital bone and terminating at the jugular foramen..$^{20}$ The anterior margin of the sulcus is more prominent than the posterior margin. Moreover, the anterior lip has three parts: lateral, junctional (with angulation) and medial; the first section is formed by the mastoid part of the temporal bone, whereas the other two are formed by the jugular process of the occipital bone. ${ }^{20}$ In the present case, an osseous bridge over the right sigmoid sulcus in the posterior cranial fossa connected the angulation of the anterior lip with the posterior lip of the sigmoid sulcus. The presence of an osseous bridge over the terminal part of the right sigmoid sinus may be due to the ossification of a thickened dura mater at the angulation. ${ }^{20}$ In the authors' opinion, this bridge could compress the sigmoid sinus and cause fatal thrombosis of the sigmoid sinus or IJ vein. Thrombosis of the sigmoid sinus or sigmoid jugular complex would block the drainage of the cerebral veins into the IJ vein; this could raise the intracranial pressure, which might subsequently cause epileptic seizures..$^{21}$ Thus, in cases of epileptic seizures of unknown aetiology, IJ vein compression by an additional belly of the ST muscle should be considered.

The presence of a LGT muscle along with a variation of the ST muscle might be due to the improper stratification of the embryological primordial muscle sheet. Since the LGT muscle and other infrahyoid muscles are derived from a common source, any variation in the infrahyoid muscle group may accompany the occurrence of the LGT muscle. Kim et al. reported a rare finding of a duplicated omohyoid muscle with the occurrence of a LGT 
muscle; the LGT muscle rose from the hyoid bone and inserted into the sheath on the anterior margin of the right lobe of the thyroid gland. ${ }^{5}$ According to Mori's classification system, this is categorised as a hyoglandularis muscle. ${ }^{22}$ This variation should be considered during surgeries to avoid iatrogenic injuries to the neurovascular structures supplying the LGT muscle. The embryological origin of a LGT muscle is under debate. Lehr opined that this muscle might be a derivative of the cricothyroid muscle. ${ }^{23}$ Eisler et al. also theorised that this muscle might be derived from the cricothyroid, thyrohyoid and inferior constrictor muscles of the pharynx. ${ }^{24} \mathrm{Kim}$ et al. suggested that the development of a LGT muscle is genetic and may be similar to the development of the omohyoid muscle. ${ }^{5}$

\section{Conclusion}

A unique variation was noted in a cadaver involving the insertion of an anomalous lateral belly of the ST muscle which passed between the IJ vein and IC artery for a short distance before insertion and lay between the glossopharyngeal and hypoglossal nerves near its termination. Infrahyoid muscles may show variations due to the improper stratification of the primordial muscle sheet; this theory is supported by the presence of a LGT muscle along with an anomalous lateral belly, as seen in this case. Knowledge of possible variations of the infrahyoid muscles is necessary in order to avoid iatrogenic trauma during surgery as well as to evaluate compression of the IJ vein in cases of idiopathic seizures.

\section{References}

1. Humphry GM. Lectures on the varieties in the muscles of man: Delivered at the Royal College of Surgeons of England. Br Med J 1873; 2:51-4. doi: 10.1136/bmj.2.655.51.

2. Standring S. Gray's Anatomy: The anatomical basis of clinical practice, 40th ed. Atlanta Georgia, USA: Churchill Livingstone, 2008. P. 442

3. Bergman RA, Afifi AK, Miyauchi R. Omohyoideus, sternohyoideus, thyrohyoideus, sternothyroideus,(infrahyoidmuscles). From: www.anatomyatlases.org/AnatomicVariants/Muscular System/Text/O/14Omohyoideus.shtml Accessed: Jun 2016

4. Nayak SR, Rai R, Krishnamurthy A, Prabhu LV, Potu BK. An anomalous belly of sternothyroid muscle and its significance. Rom J Morphol Embryol 2009; 50:307-8.

5. Kim DI, Kim HJ, Park JY, Lee KS. Variation of the infrahyoid muscle: Duplicated omohyoid and appearance of the levator glandulae thyroideae muscles. Yonsei Med J 2010; 51:984-6. doi: $10.3349 /$ ymj.2010.51.6.984.

6. Keibel F, Mall FP. Manual of Human Embryology 12: The ventrolateral muscles of the neck. From: embryology.med. unsw.edu.au/embryology/index.php/Book___Manual_of_ Human_Embryology_12\#The_Ventrolateral_Muscles_of_the Neck Accessed: Jun 2016.
7. Dastjerdi A, Robson L, Walker R, Hadley J, Zhang Z, Rodriguez-Niedenführ $M$, et al. Tbx1 regulation of myogenic differentiation in the limb and cranial mesoderm. Dev Dyn 2007; 236:353-63. doi: 10.1002/dvdy.21010.

8. Zhang Z, Huynh T, Baldini A. Mesodermal expression of Tbx1 is necessary and sufficient for pharyngeal arch and cardiac outflow tract development. Development 2006; 133:3587-95. doi: $10.1242 /$ dev.02539.

9. Tripathy PR, Preetam C. Unilateral absence of the sternohyoid muscle with contralateral tendinous belly of superior belly of omohyoid muscle: A very rare form of infrahyoid muscle variation. Int J Anat Res 2015; 3:1302-5. doi: 10.16965/ ijar.2015.216.

10. Wood J. Variations in human myology observed during the Winter Session of 1865-66 at King's College, London. Proc R Soc Lond 1867; 15:229-44. doi: 10.1098/rspl.1866.0054.

11. Thakur A, A B, Parmar SK. Multiple variation in neck musculature and their surgical implications. Int J Anat Var $2011 ; 4: 171-3$.

12. Simka M, Majewski E, Fortuna $M$, Zaniewski M. Internal jugular vein entrapment in a multiple sclerosis patient. Case Rep Surg 2012; 2012:293568. doi: 10.1155/2012/293568.

13. Patra P, Gunness TK, Robert R, Rogez JM, Heloury Y, Le Hur PA, et al. Physiologic variations of the internal jugular vein surface, role of the omohyoid muscle, a preliminary echographic study. Surg Radiol Anat 1988; 10:107-12. doi: $10.1007 /$ BF02307818.

14. Jayaraman MV, Boxerman JL, Davis LM, Haas RA, Rogg JM. Incidence of extrinsic compression of the internal jugular vein in unselected patients undergoing CT angiography. ANJR Am J Neuroradiol 2012; 33:1247-50. doi: 10.3174/ajnr.A2953.

15. Mehta V, Gupta V, Arora J, Yadav Y, Suri RK, Rath G. Bilateral bipartite origin of the posterior belly of digastric muscle: A clinico-anatomical appraisal. Anat 2011; 5:44-7. doi: 10.2399/ ana.09.042.

16. Zaniewski M, Simka M. Biophysics of venous return from the brain from the perspective of the pathophysiology of chronic cerebrospinal venous insufficiency. Rev Recent Clin Trials 2012; 7:88-92. doi: 10.2174/157488712800100288.

17. Ball E, Morris-Stiff G, Coxon M, Lewis MH. Internal jugular vein thrombosis in a warfarinised patient: A case report. J Med Case Rep 2007; 1:184. doi: 10.1186/1752-1947-1-184.

18. Handley TP, Miah MS, Majumdar S, Hussain SS. Collet-Sicard syndrome from thrombosis of the sigmoid-jugular complex: A case report and review of the literature. Int J Otolaryngol 2010; 2010:203587. doi:10.1155/2010/203587.

19. Hofmann E, Behr R, Neumann-Haefelin TN, Schwager K. Pulsatile tinnitus: Imaging and differential diagnosis. Dtsch Arztebl Int 2013; 110:451-8. doi: 10.3238/arztebl.2013.0451.

20. Singh P, Tuli A, Choudhry R, Dhall U, Makwane UK. Morphology and imaging of bony projections on sigmoid sulcus with clinical implication. Surg Radiol Anat 2004; 26:46-50. doi: 10.1007/s00276-003-0173-3.

21. Alvis-Miranda HR, Milena Castellar-Leones S, Alcala-Cerra G, Rafael Moscote-Salazar L. Cerebral sinus venous thrombosis. J Neurosci Rural Pract 2013; 4:427-38. doi: 10.4103/0976-31 47.120236 .

22. Mori M. Statistics on the musculature of the Japanese. Okajimas Folia Anat Ipn 1964; 40:195-300. doi: 10.2535/ ofaj1936.40.3_195.

23. Lehr RP Jr. Musculus levator glandulae thyroideae: An observation. Anat Anz 1979; 146:494-6.

24. Eisler P, Der M. Levator glandulae thyroideae und verwandte praelaryngeale Muskelbildungen. Anat Anz 1900; 17:183-9. 